\title{
AVALIAÇÃO DA ATENÇÃO PRIMÁRIA NO CONTROLE DA HANSENÍASE: UM ESTUDO REALIZADO EM UM MUNICÍPIO HIPERENDÊMICO DO NORDESTE BRASILEIRO
}

\author{
Marcos Alan Sousa Barbosa*1, Marilena Maria de Souza², Rayanne de Sousa Barbosa3, \\ Rogéria Mônica Seixas Xavier de Abreu $^{4}$ and Jardel Rhodes Carolino de Oliveira ${ }^{5}$ \\ ${ }_{1}^{1}$ Unidade Acadêmica de Ciências da Vida, Universidade Federal de Campina Grande, Cajazeiras, Brasil \\ ${ }^{2}$ Escola Técnica de Saúde de Cajazeiras, Universidade Federal de Campina Grande, Cajazeiras, Brasil \\ ${ }^{3}$ Departamento de Enfermagem, Centro Universitário Vale do Salgado, Icó, Brasil \\ ${ }^{4}$ Mestrado em Sistemas Agroindustriais, Universidade Federal de Campina Grande, Pombal, Brasil \\ 5Pós-Graduação em Educação Continuada, Centro Universitário de João Pessoa, João Pessoa, Brasil
}

\section{ARTICLE INFO}

\section{Article History:}

Received $20^{\text {th }}$ February, 2021

Received in revised form

$26^{\text {th }}$ March, 2021

Accepted $04^{\text {th }}$ April, 2021

Published online $30^{\text {th }}$ May, 2021

Key Words:

Hanseníase, Atenção Primária à Saúde, Avaliação em Saúde, Gestão.

*Corresponding author:

Marcos Alan Sousa Barbosa

\begin{abstract}
A hanseníase continua sendo um problema de saúde pública no Brasil, ainda apresentando clusters de alta detecção da doença em seu território. Uma dessas áreas abrange o município de Cajazeiras, na Paraíba, que apresenta índices de alta endemicidade para a doença. Logo, objetivase avaliar o desempenho desse município na atenção à hanseníase por meio de um instrumento de avaliação do grau de orientação dos serviços da Atenção Primária à Saúde na realização das ações de controle da hanseníase. Para isso, desenhou-se um estudo avaliativo executado nas unidades de saúde a partir da aplicação desse instrumento. Logo, percebeu-se que o município possuía graves iniquidades nos atributos "acesso" e "orientação profissional". Os altos desvios padrões indicaram também que havia aspectos negativos sendo mascarados dentro de atributos com alta avaliação, tais como: ausência de contrarreferência e dificuldade de encaminhamento para especialistas, pouco suporte de uma equipe de supervisão municipal, insuficiente conhecimento epidemiológico da doença no município, ausência de um ambulatório de referência na cidade. Assim, a aplicação desse instrumento permitiu uma visão pormenorizada das áreas em que as unidades e a gestão municipal têm falhado, permitindo a correção desses erros e o aprimoramento da atenção ao paciente com hanseníase.
\end{abstract}

Copyright (C) 2021, Marcos Alan Sousa Barbosa et al. This is an open access article distributed under the Creative Commons Attribution License, which permits unrestricted use, distribution, and reproduction in any medium, provided the original work is properly cited.

Citation: Marcos Alan Sousa Barbosa, Marilena Maria de Souza, Rayanne de Sousa Barbosa et al. "Avaliação da atenção primária no controle da hanseníase: um estudo realizado em um município hiperendêmico do nordeste brasileiro”, International Journal of Development Research, 11, (05), 4731847321.

\section{INTRODUCTION}

A hanseníase é uma doença infecciosa crônica com sintomas dermatoneurológicos causada pelo Mycobacterium leprae, sendo conhecida por possuir altainfectividade e baixa patogenicidade (Brasil, 2017). Segundo a World Health Organization (2013), a magnitude e abrangência da hanseníase a tornaram um grave problema de saúde pública. Em 2012, o Brasil notificou 33 mil casos novos da doença, ocupando o segundo lugar no ranking mundial, ficando atrás apenas da Índia, com cerca de 130 mil casos. As principais políticas de saúde desenvolvidas pelo Ministério da Saúde (MS) a fim de reduzir esses níveis endêmicos de hanseníase envolvem a criação de uma rede de atenção com a integração das ações de controle da doença $(\mathrm{ACH})$, a saber: detecção oportuna de novos casos; tratamento com o esquema poliquimioterápico; prevenção de incapacidades e vigilância dos contatos domiciliares. Essas ações são executadas primordialmente na atenção primária à saúde (APS) e a manutenção da atenção especializada acontece nos níveis secundário e terciário em razão do potencial incapacitante da doença (Brasil, 2016). Com essa integração, a Estratégia de Saúde da Família (ESF) deve estar organizada para oferecer as ações de controle da doença de acordo com os atributos da APS, não só em relação ao acesso, diagnóstico/tratamento e orientação para a comunidade, mas também devem estar intimamente relacionadas com 
as demais dimensões da APS como porta de entrada, atendimento continuado, integralidade dos serviços, coordenação e enfoque na família (Lanza, 2014). Contudo, mesmo com a formulação das ACH, um estudo de geoprocessamento realizado no país identificou vinte e seis clusters (aglomerados/áreas de concentração) com altas taxas de detecção para hanseníase. A Paraíba (PB) está incluída nessesclusters, sendo a sua área de maior endemicidade compreendida na mesorregião do sertão paraibano, mais especificamente nas microrregiões de Cajazeiras, Itaporanga, Catolé do Rocha, Piancó e Serra do Teixeira (Brasil, 2010). Dentre as cidades supracitadas, o município de Cajazeiras apresenta coeficientes alarmantes da doença. Verificou-se que, durante os anos de 2010 a 2018, todas as taxas de detecção de novos casos da doença situavam-se na faixa considerada pelos parâmetros do MS como hiperendêmica (acima de 40,00/100.000 hab.), excetuando-se o ano de 2014 e 2016 quando o coeficiente foi considerado de alta endemicidade $(20,00$ a 39,99/100.000 hab.) (Brasil, 2019).

Assim, a avaliação da implementação e condução das $\mathrm{ACH}$ em cidades de alta endemicidade é imprescindível no diagnóstico e correção de falhas, sendo um importante instrumento para aperfeiçoar a atenção prestada ao paciente com hanseníase. E, embora existam indicadores epidemiológicos que permitam a análise do $\mathrm{ACH}$, eles não avaliam diretamente a execução dessas políticas na APS com base nas suas atribuições, impossibilitando uma visão pormenorizada do problema. Para isso, este estudo objetivou avaliar o desempenho do município de Cajazeiras-PB na atenção à hanseníase por meio de um instrumento de avaliação do grau de orientação dos serviços da atenção primária à saúde na realização das ações de controle da hanseníase previstas pelo MS.

\section{MATERIAL E MÉTODOS}

Trata-se de uma pesquisa avaliativa de natureza quantitativa, tendo sido realizada no município de Cajazeiras-PB, situado na extremidade ocidental do estado da Paraíba, na região Nordeste do país.O município apresenta um coeficiente de detecção de novos casos para hanseníase considerado como hiperendêmico, além de estar situada no maior cluster de aglomeração da doença do estado da Paraíba (Brasil, 2016; Brasil, 2019). A pesquisa foi aplicada em todas as Unidades Básicas de Saúde (UBS) de Cajazeiras-PB, a fim de que se tenha um retrato fidedigno de como as $\mathrm{ACH}$ estão sendo conduzidas no município. Nesse cenário, os enfermeiros foram escolhidos como os representantes das suas respectivas unidades para preenchimento do instrumento, visto que esse profissional se comporta como um coordenador nos cuidados realizados em APS, recebendo devolutivas dos demais profissionais e articulando as suas atribuições. Além disso, são reconhecidos pelos próprios usuários como os profissionais mais ativos na atenção voltada ao paciente com hanseníase (Lanzoni et al., 2016; Cabral et al., 2016; Ribeiro, 2017). A população foi constituída por enfermeiros que concordaram em participar do estudo e que atenderam ao critério de inclusão, sendo ele: enfermeiros que atuem em unidades de APS do município de Cajazeiras-PB. Já os critérios de exclusão foram: profissionais que não estiverem presentes na unidade no dia do agendamento da coleta de dados, bem como aqueles que estiverem de férias regulamentares, licença médica e licença maternidade.

A fim de que o objetivo fosse atingido, foi usado o "Instrumento de avaliação do desempenho da atenção primária nas ações de controle da hanseníase- versão enfermeiros" que foi elaborado, validado e aplicado por Lanza (2014) e adaptado pelos autores dessa pesquisa, tendo sido elaborado a partir das ações de controle da hanseníase preconizadas pelo MS para serem desempenhadas na APS. A sua estrutura foi elaborada a partir do formato utilizado no instrumento Primary Care Assessment Tool (PCAT) adotado como referência pelo MS (Brasil, 2010). Os 76 itens do instrumento foram alocados dentro de oito construtos que foram formulados a partir dos documentos oficiais do Sistema Único de Saúde para a atenção à hanseníase na APS. São eles: porta de entrada, acesso, atendimento continuado, integralidade dos serviços disponíveis e prestados, coordenação, orientação familiar, orientação comunitária e orientação profissional, correspondendo aos atributos da APS nas ACH. O instrumento foi aplicado através de uma entrevista, na qual o entrevistado respondeu os itens utilizando a escala de Likert, com as mesmas opções de respostas do PCATool-Brasil: 1 (com certeza não); 2 (provavelmente não); 3 (provavelmente sim); 4 (com certeza sim); 9 (não sei/não lembro). Para o cálculo dos escores, foi utilizada a mesma metodologia do PCAT-Brasil, possibilitando a produção de escores e médias para cada atributo da APS, um escore geral sobre a qualidade da atenção da hanseníase em cada UBS e um escore global para o município de Cajazeiras (Brasil, 2010).

O serviço é considerado de alta orientação para as $\mathrm{ACH}$ preconizadas pelo MS quando o escore é maior ou igual que 6,6 e de baixa orientação quando é menor que 6,6. Além disso, as medidas de dispersão devem ser analisadas a fim de notar a distribuição dos dados ao redor do escore, evidenciando se a dispersão é de caráter heterogêneo ou homogêneo. A coleta dos dados com os profissionais foi realizada no ambiente de trabalho, marcada em um horário de acordo com a disponibilidade de cada profissional, em um local reservado para não ocorrerem interrupções e garantir a privacidade do informante. Na realização da aplicação, se o participante do estudo não tiver atendido um caso de hanseníase na atual unidade de saúde/microárea, alguns itens do instrumento não serão respondidos, sendo codificados como "88 - não se aplica (missings)". Vale salientar que, todos os sujeitos só participaram do estudo mediante a assinatura do Termo de Consentimento Livre e Esclarecido (TCLE), atendendo às determinações da Resolução 466/2012 (Brasil, 2012), que estabelece as diretrizes e normas regulamentadoras de pesquisas envolvendo seres humanos.

\section{RESULTADOS E DISCUSSÕES}

Do total de 24 enfermeiros pré-estabelecidos como população do estudo, duas estavam de licença maternidade e um não compareceu ao local agendado para a entrevista mesmo após múltiplas tentativas, sendo subtraídos da população por atenderem aos critérios de exclusão estabelecidos previamente. Logo, a amostra final foi de 21 enfermeiros e unidades representadas. A análise das médias demonstrou que há dois atributos situados abaixo de 6,6. Assim, os atributos acesso (média $=5$ ) e orientação profissional (média $=5,3$ ) foram considerados de baixa orientação para a atenção ao paciente com hanseníase em Cajazeiras-PB (Tabela 01). A análise dos escores dos itens que constituem o atributo acesso evidenciou que as fragilidades desse atributo estão relacionadas, principalmente, à ausência de telefone nas unidades de saúde (item D2; média=0), ao horário de funcionamento das unidades, que ficam abertas apenas até às 17 horas (item D1; média $=0$ ), à dificuldade de acesso à UBS (item D3; média $=6,3)$ e à necessidade dos usuários se ausentarem do trabalho para serem atendidos na UBS (item D5; média=6,1), resultado que está diretamente relacionado ao horário de funcionamento da unidade, que é semelhante ao horário comercial. Campos et al. (2014) realizou um estudo que apontou a insatisfação dos usuários em relação ao acesso e à acessibilidade das UBSs. A territorialização ineficaz ainda permite que grandes regiões permaneçam descobertas e/ou distantes da UBS, além da dificuldade em oportunizar outros momentos de atendimento fora do horário comercial, dificultando o processo de cuidado ao paciente com hanseníase. Por sua vez, a análise dos escores dos itens que constituem o atributo orientação profissional evidenciou que as fragilidades desse atributo estão relacionadas, principalmente, à ausência de treinamentos frequentes de hanseníase no município (item J3; média=1). Desse modo, é de fundamental importância que os profissionais sejam continuamente capacitados para desempenharem as ACH na APS adequadamente. Justifica-se então a limitação dos gestores na utilização das portarias do MS para o planejamento da educação continuada desses profissionais. Tal omissão constrói um ciclo vicioso alimentado pela falta de informações e de alinhamento com a política nacional de hanseníase resultando numa atenção desarticulada à necessidade desses pacientes (Lanza, 2014). 
Tabela 1. Descrição das médias dos atributos por UBS e das médias gerais e medidas de dispersão dos atributos em Cajazeiras-PB

\begin{tabular}{|c|c|c|c|c|c|c|c|c|}
\hline Nome da Unidade Básica de Saúde & 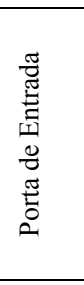 & $\begin{array}{l}0 \\
\text { d } \\
\text { d } \\
\text { \& }\end{array}$ & 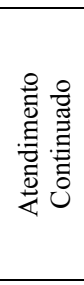 & 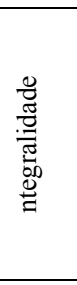 & 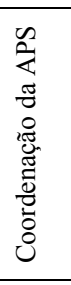 & 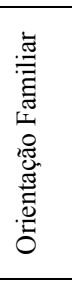 & 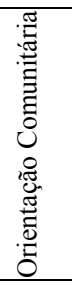 & 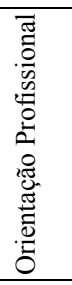 \\
\hline Casas Populares & 10 & 4,4 & 9,7 & 9,8 & 6,8 & 10 & 9,3 & 5,5 \\
\hline Mutirão I & 9,3 & 5,9 & 8,8 & 8,8 & 5,9 & 9,6 & 8,7 & 6,6 \\
\hline Mutirão II & 8,3 & 5,5 & 8,8 & 9,4 & 6,6 & 9,6 & 9,3 & 5,5 \\
\hline Amélio Estrela & 10 & 7 & 9,7 & 9,4 & 6,6 & 10 & 9,3 & 5,5 \\
\hline Francisco Valiomar & 6,6 & 5,5 & 8,8 & 9,4 & 6,8 & 7,7 & 9,3 & 5,5 \\
\hline Bela Vista & 5,8 & 5,9 & 8,2 & 8,4 & 7,1 & 9,6 & 7,3 & 4,4 \\
\hline $\mathrm{Fco}^{\mathrm{a}}$ Gonçalves & 9,2 & 2,6 & 10 & 9,3 & 6,5 & 10 & 7,3 & 4,4 \\
\hline $\mathrm{M}^{\mathrm{a}}$ José de Jesus & 9,2 & 5,5 & 9,4 & 9,1 & 7,3 & 9,6 & 10 & 5,5 \\
\hline Esperança & 7,5 & 6,3 & 6,7 & 7,5 & 6 & 7,8 & 8,7 & 6,6 \\
\hline Simão de Oliveira & $*$ & 4,1 & * & 5,9 & 7,3 & $*$ & 4 & 3,3 \\
\hline José Lopes de Lira & 6,6 & 3,3 & 8,9 & 9,4 & 6,6 & 9,2 & 4,6 & 5,5 \\
\hline João Bosco & 8,3 & 5,9 & 7,9 & 9,4 & 7,6 & 10 & 10 & 6,6 \\
\hline Dr. Vital Rolim & 9,2 & 5,2 & 9,4 & 9,8 & 8 & 10 & 8,7 & 5,5 \\
\hline José Leite Rolim & 9,2 & 5,5 & 9,7 & 9,4 & 6,2 & 10 & 8,7 & 6,6 \\
\hline Francisco Nunes & 8,3 & 5,5 & * & 8,8 & 5,9 & $*$ & 5,3 & 6,6 \\
\hline Patamute & 10 & 5,5 & 10 & 9,7 & 7 & 8,8 & 8 & 4,4 \\
\hline Catolé & 8,3 & 5,9 & 7,6 & 10 & 8,2 & 8,8 & 9,3 & 5,5 \\
\hline Higino Dias Moreira & 7,5 & 4,1 & 8,2 & 9 & 6,6 & 5,9 & 6 & 6,6 \\
\hline Tancredo Neves & 10 & 3,3 & 8,2 & 8,8 & 8,7 & 9,6 & 6,6 & 5,5 \\
\hline São Francisco & 10 & 2,2 & 10 & 9,1 & 6,8 & 5,2 & 6,6 & 6,6 \\
\hline Nilson Lopes & 7,5 & 6,6 & 7,9 & 9,1 & 7,6 & 10 & 4 & 0 \\
\hline Média Geral & 8,6 & 5 & 8,8 & 9 & 6,9 & 9 & 7,6 & 5,3 \\
\hline Desvio Padrão & $\pm 1,2$ & $\pm 1,3$ & $\pm 0,9$ & $\pm 0,8$ & $\pm 0,7$ & $\pm 1,4$ & $\pm 1,9$ & $\pm 1,5$ \\
\hline Coeficiente de Variação (\%) & 14,8 & 26,2 & 10,6 & 9,8 & 10,7 & 15,6 & 25,5 & 28,6 \\
\hline Mediana & 8,7 & 5,5 & 8,8 & 9,3 & 6,8 & 9,6 & 8,7 & 5,5 \\
\hline
\end{tabular}

* Escore não calculado devido $>50 \%$ de missings Fonte: Dados do Estudo

Tabela 02. Escore geral, medidas de dispersão e mediana dos atributos do instrumento por UBS de Cajazeiras-PB

\begin{tabular}{|l|c|c|c|c|}
\hline & Escore Geral & Desvio Padrão & CV* & Mediana \\
\hline Casas Populares & 8,2 & $\pm 2,27$ & $27,6 \%$ & 9,5 \\
\hline Mutirão I & 7,9 & $\pm 1,54$ & $19,4 \%$ & 8,75 \\
\hline Mutirão II & 7,9 & $\pm 1,74$ & $22 \%$ & 8,55 \\
\hline Amélio Estrela & 8,4 & $\pm 1,78$ & $21,1 \%$ & 9,35 \\
\hline Francisco Valiomar & 7,4 & $\pm 1,59$ & $21,4 \%$ & 7,25 \\
\hline Bela Vista & 7,1 & $\pm 1,67$ & $23,5 \%$ & 7,2 \\
\hline Francisco Gonçalves & 7,4 & $\pm 2,75$ & $37,1 \%$ & 8,25 \\
\hline $\mathrm{M}^{\mathrm{a}}$ José de Jesus & 8,2 & $\pm 1,84$ & $22,4 \%$ & 9,15 \\
\hline Esperança & 7,1 & $\pm 0,89$ & $12,5 \%$ & 7,1 \\
\hline Simão de Oliveira & 4,9 & $\pm 1,64$ & $33,4 \%$ & 4,1 \\
\hline José Lopes de Lira & 6,8 & $\pm 2,26$ & $33,2 \%$ & 6,6 \\
\hline João Bosco & 8,2 & $\pm 1,52$ & $18,5 \%$ & 8,1 \\
\hline Dr. Vital Rolim & 8,2 & $\pm 1,88$ & $22,9 \%$ & 8,95 \\
\hline José Leite Rolim & 8,1 & \pm 1.77 & $21,8 \%$ & 8,95 \\
\hline Francisco Nunes & 6,7 & $\pm 1,46$ & $21,7 \%$ & 6,6 \\
\hline Patamute & 8,4 & $\pm 2,12$ & $25,2 \%$ & 8,4 \\
\hline Carlindo Francisco Soares & 8,2 & $\pm 1,57$ & $19,1 \%$ & 8,25 \\
\hline Higino Dias Moreira & 6,6 & $\pm 1,51$ & $22,87 \%$ & 6,6 \\
\hline Tancredo Neves & 7,6 & $\pm 2,28$ & $30 \%$ & 8,45 \\
\hline São Francisco & 7,1 & $\pm 2,64$ & $37,1 \%$ & 6,7 \\
\hline Nilson Lopes & 6,5 & $\pm 3,19$ & $49 \%$ & 7,55 \\
\hline CAJAZEIRAS & 7,5 & \pm 2 & $26,6 \%$ & 7,7 \\
\hline
\end{tabular}

*CV: Coeficiente de Variação Fonte: Dados do Estudo

O coeficiente de variação (CV) é uma variável que analisa a dimensão do desvio padrão, determinando, dessa forma, se a distribuição dos dados é mais homogênea ou heterogênea ao redor da média. Gomes (1985) apresentou a formulação dos parâmetros que definem o coeficiente de variação como baixo $(<10 \%)$, médio $(10 \%-20 \%)$, alto $(>20 \%)$ e muito alto $(>30 \%)$. Logo, tendo em vista a Tabela 01 , é possível observar que quatro atributos (porta de entrada, atendimento continuado, coordenação e orientação familiar) possuem CV médios e três altos (acesso, orientação comunitária e orientação profissional), evidenciando uma dispersão significativa dos dados ao redor da média.
Assim, embora a maioria dos atributos apresentem médias satisfatórias, a alta dispersão demonstra que alguns aspectos foram avaliados negativamente dentro dos atributos, sendo a sua discriminação de alta importância para a identificação de problemas pontuais e aperfeiçoamento da atenção ao paciente com hanseníase. Através do cálculo da média dos itens foi possível identificar alguns desses aspectos avaliados negativamente, a saber:

- $\quad$ Atendimento para problemas de saúde mental (item F12 de Integralidade; média $=5$ ). 
- Colaboração de uma equipe de supervisão municipal de hanseníase na realização das ações de controle da doença (item G7 de Coordenação; média 4).

- Problema de saúde relacionado à hanseníase (ex: neurite, reações medicamentosas, reações hansênicas) e a dificuldade para encaminhar ao especialista (item G8 de Coordenação; média $=5,3$ ).

- Contrarreferência do paciente de hanseníase acompanhada por formulário contendo as informações a respeito do atendimento prestado e das condutas para o seguimento do paciente no serviço de origem (item G11 de Coordenação; média 1,7).

- Disponibilidade de serviços de neurologia no município (item G19 de Coordenação; média=4).

O escore geral do município de Cajazeiras foi de 7,5, configurado como de alta orientação para as $\mathrm{ACH}$. Porém ela não permite uma visão pormenorizada das iniquidades da atenção ao paciente afetado pela hanseníase na cidade. Tal raciocínio é provado através da análise do desvio padrão, categorizado como de alta dispersão, demonstrando que existem aspectos fortemente negativos sendo mascarados por aspectos avaliados como positivos (Tabela 02 ).

De um modo geral, todas as UBSs avaliadas até o presente momento apresentaram escores gerais (EG) acima de 6,6, com exceção da UBS Simão de Oliveira $(E G=4,9)$ e Nilson Lopes $(E G=6,5)$. Embora esse dado signifique que há alta orientação dos serviços para a hanseníase na maior parte das UBSs, deve-se notar que os maiores escores gerais foram da UBS Amélio Estrela e da UBS Patamute $(E G=8,4)$, estando ainda distante de médias mais satisfatórias, principalmente em se tratando de um município hiperendêmico para a doença. Vale salientar também o altíssimo CV das UBSs Francisco Gonçalves, Simão de Oliveira, José Lopes de Lira, Tancredo Neves, São Francisco e Nilson Lopes, indicando uma heterogeneidade da dispersão das médias e evidenciando que graves problemas pontuais estão sendo mascarados pela média satisfatória apresentada, confirmando a necessidade de avaliar a média de cada item do instrumento.

\section{CONCLUSÕES}

A hanseníase continua sendo um problema de saúde pública no Brasil. Embora tenha havido uma clara diminuição dos coeficientes de detecção na maior do país, ainda existem clusters com altas taxas de deteç̧ão da doença. O município de Cajazeiras-PB exemplifica esse fenômeno, uma vez que ainda apresenta coeficientes de alta endemicidade para a doença. A avaliação como componente da gestão em saúde tem hoje um reconhecimento. O propósito fundamental é dar suporte aos processos decisórios no âmbito do SUS, devendo subsidiar a identificação de problemas e a reorientação de ações e serviços desenvolvidos, avaliar a incorporação de novas práticas sanitárias na rotina dos profissionais, bem como mensurar o impacto das ações implementadas pelos serviços e programas sobre o estado de saúde da população (Vieira-da-silva \& Furtado, 2020). Em relação ao combate à hanseníase, as próprias políticas de eliminação da doença preconizam o monitoramento e a avaliação como ferramentas de melhoria do programa e de promoção da gestão, sendo imprescindível a sua incorporação na rotina dos planejadores, gestores e executores do programa (Brasil, 2016). Esse estudo avaliativo proporcionou evidências que indicam iniquidades nos atributos "acesso" e "orientação profissional" no município de Cajazeiras-PB, ambos avaliados com escores que indicam baixa orientação às $\mathrm{ACH}$. Logo, é necessário possibilitar melhorias no acesso e na acessibilidade a essas ações e investir na educação continuada dos profissionais que as executam. Além disso, outros aspectos foram avaliados negativamente dentro de atributos que, no geral, foram considerados de alta orientação para as ACH. O emprego e análise dos desvios padrão propiciou essa investigação que permitiu a identificação de problemas pontuais que necessitam de intervenção, tais como: carência de suporte psicológico aos pacientes com hanseníase, pouca participação da equipe de supervisão municipal da realização das $\mathrm{ACH}$, dificuldades de encaminhamento ao especialista na vigência de complicações, ausência de contrarreferência da atenção secundária e terciária, pouco conhecimento do estado epidemiológico da hanseníase nas microáreas, carência de serviços de neurologia no município e de um ambulatório de referência para a doença.

Ademais, esse estudo apresentou dados que auxiliam na gestão das $\mathrm{ACH}$ da APS do município, podendo ser usadas por três grupos distintos: o primeiro deles é a equipe municipal de saúde, que deve refletir sobre aspectos relacionados à gestão e à implementação de estratégias municipais que visem amenizar essas iniquidades; o segundo grupo são os próprios profissionais e coordenadores da APS que, com os escores de suas UBSs, podem trabalhar estrategicamente para a correção desses agravos, proporcionando uma atenção mais adequada ao paciente com hanseníase; e o terceiro é composto por pesquisadores e gestores que, no desejo de avaliar as ACH em seus municípios, podem replicar a metodologia desse estudo à nível local.

\section{REFERÊNCIAS}

Brasil. Ministério da saúde 2010. Manual do instrumento de avaliação da atenção primária à saúde: primary care

assessment tool pcatool. Secretaria de Atenção em Saúde. Departamento de Atenção Básica.

Brasil.Ministério da saúde 2010. Portaria n. 594 de 29 de outubro de 2010. Aprova o Serviço de Atenção Integral em Hanseníase. República Federativa do Brasil.

Brasil. Ministério da saúde 2016. Diretrizes para vigilância, atenção e eliminação da hanseníase como problema de saúde pública 60 . Secretaria de Vigilância em Saúde.

Brasil. Ministério da saúde 2016. Guia Prático Sobre a Hanseniase 1st ed.. Secretaria de Vigilância em Saúde.

Brasil. Ministério da saúde 2019. Hanseníase - Notificações Registradas: banco de dados. Sistema de informação de

Agravos de Notificação - SINAN. Retrieved April 18, 2019, from http://tabnet.datasus.gov.br/cgi/dhx.exe?han/hantfpb.def

Brasil. Ministério da Saúde. 2012. Resolução $n^{\circ} 466$ de 12 de dezembro de 2012 1. Conselho Nacional de Saúde.

Comissão Nacional de Ética em Pesquisa. http://conselho.saude.gov.br/resolucoes/2012/Reso466.pdf

Cabral, CVS, de Oliveira Costa, MA, de Oliveira Lima, RB, dos Santos Silva, J., da Costa Cabral, L., \& Rocha, N. M. C. 2016. O papel do enfermeiro na prevenção de incapacidades e deformidades no portador de hanseníase. Revista Interdisciplinar, 92, 168-177.

Campos, RTO, Ferrer, AL, Gama, CAPD, Campos, GWDS, Trapé, TL, \& Dantas, DV 2014. Avaliação da qualidade do acesso na atenção primária de uma grande cidade brasileira na perspectiva dos usuários. Saúde em Debate, 38, 252-264.

Gomes, P. 1985. Curso de Estatística Experimental 1st ed.. Nobel.

Lanza, FM 2014. Avaliação da atenção primária no controle da hanseníase: validação de instrumentos e análise do desempenho de municípios endêmicos do Estado de Minas Gerais [Tese]. Belo Horizonte: Doutorado; 2014. 311 s. Curso de Enfermagem, Universidade Federal de Minas Gerais.

Lanzoni Melo, Meirelles, BHS, \& Cummings, G. 2016. Práticas de liderança do enfermeiro na atenção básica à saúde: uma teoria fundamentada nos dados. Texto \& Contexto Enfermagem, 254, 19.

Ribeiro, MDA, da Silva Castillo, I., Silva, JCA, \&Oliveira, SB 2017. A visão do profissional enfermeiro sobre o tratamento da hanseníase na atenção básica. Revista Brasileira em Promoção da Saúde, 302.

Vieira-da-Silva, LM, \& Furtado, JP 2020. A avaliação de programas de saúde: continuidades e mudanças. Cadernos de Saúde Pública..

World Health Organization. 2013. Global leprosy: update on 2012 situation. Wkly Epidemiol, 365-79. 